\title{
Validation of the animal model of bipolar disorder induced by Ouabain: face, construct and predictive perspectives
}

\author{
Samira S. Valvassori ${ }^{1}$, Gustavo C. Dal-Pont ${ }^{1}$, Wilson R. Resende', Roger B. Varela', Jéssica Lopes-Borges ${ }^{1}$,
} José Henrique Cararo ${ }^{1}$ and João Quevedo (1) 1,2,3,

\begin{abstract}
A particular challenge in the development of a bipolar disorder (BD) model in animals is the complicated clinical course of the condition, characterized by manic, depressive and mixed mood episodes. Ouabain (OUA) is an inhibitor of $\mathrm{Na}^{+} /$ $\mathrm{K}^{+}$-ATPase enzyme. Intracerebroventricular (ICV) injection of this drug in rats has been regarded a proper model to study BD by mimic specific manic symptoms, which are reversed by lithium (Li), an important mood stabilizer drug. However, further validation of this experimental approach is required to characterize it as an animal model of BD, including depressive-like behaviors. The present study aimed to assess manic- and depressive-like behaviors, potential alteration in the hypothalamic-pituitary-adrenal (HPA) system and oxidative stress parameters after a single OUA ICV administration in adult male Wistar rats. Moreover, we evaluated Li effects in this experimental setting. Data show that OUA ICV administration could constitute a suitable model for BD since the injection of the drug triggered manic- and depressive-like behaviors in the same animal. Additionally, the OUA model mimics significant physiological and neurochemical alterations detected in BD patients, including an increase in oxidative stress and change in HPA axis. Our findings suggest that decreased $\mathrm{Na}^{+} / \mathrm{K}^{+}$-ATPase activity detected in bipolar patients may be linked to increased secretion of glucocorticoid hormones and oxidative damage, leading to the marked behavioral swings. The Li administration mitigated these pathological changes in the rats. The proposed OUA model is regarded as suitable to simulate BD by complying with all validities required to a proper animal model of the psychiatric disorder.
\end{abstract}

\section{Introduction}

Bipolar disorder (BD) is a psychiatric condition marked by aberrant mood swings, including mania, depression, and mixed states. Despite its significant impact in the life quality of the patients, both pathophysiology and pathogenesis of this disorder remain unclear $^{1-3}$. In this regard, animal models are an essential strategy in search of data on behavioral status, neurotransmitter systems, and mechanisms underlying a particular mental disorder ${ }^{4-6}$.

\footnotetext{
Correspondence: Samira S. Valvassori (samiravalvassori@unesc.net)

${ }^{1}$ Translational Psychiatry Laboratory, Graduate Program in Health Sciences,

University of Southern Santa Catarina (UNESC), Criciúma, SC, Brazil

${ }^{2}$ Translational Psychiatry Program, Department of Psychiatry and Behavioral

Sciences, McGovern Medical School, The University of Texas Health Science

Center at Houston (UTHealth), Houston, TX, USA

Full list of author information is available at the end of the article.
}

However, validation of an animal model for BD is difficult due to the complex clinical course of this condition ${ }^{7}$.

At present, most of the animal models for BD induces behaviors acutely mimicking a manic or depressive episode, not a combination thereof ${ }^{4,7,8}$. It is noteworthy that animal models of mental disorders should comply with the following requirements: ${ }^{9}$ Face validity, which indicates whether the model mimics the symptoms of a particular disorder; Construct validity, showing the ability of the model to simulate pathophysiological aspects of the illness; Predictive validity, which assesses whether the drugs included in therapy for the human condition can reverse the symptoms induced in the model.

The animal model of mania elicited by ouabain (OUA) - an inhibitor of $\mathrm{Na}^{+} / \mathrm{K}^{+}$-ATPase enzyme (EC 3.6.3.9), 
seems to meet these basic requirements, which renders it suitable to study certain behavioral and neurochemical aspects of $\mathrm{BD}^{10-13}$. OUA dose-dependently increases locomotor activity in rats, which is associated with maniclike behavior ${ }^{12,14,15}$. Intracerebroventricular (ICV) injection of this glycoside in rats also induces hypoactivity, which could be considered a depressive-like behavior. Such behavioral alterations are significantly prevented by administration of lithium (Li), an important mood stabi$\operatorname{lizer}^{13,16}$. However, hypoactivity is not enough to mimic a state of depression, and additional experiments are necessary to validate this model. Besides, no research group showed manic- and depressive-like behaviors in the same animal after administration of OUA. The OUA model of mania was designed according to the " $\mathrm{Na}^{+} / \mathrm{K}^{+}$ATPase hypothesis", which hints decreased activity of the enzyme as a key factor to the outbreak of manic and depressive mood episodes in $\mathrm{BD}^{14,17}$. Indeed, several papers in the literature show that $\mathrm{Na}^{+} / \mathrm{K}^{+}$-ATPase activity is decreased in bipolar patients ${ }^{17}$. In addition, ICV administration of OUA in rats induces molecular changes similar to those detected in BD patients, neurotrophic factor alterations, mitochondrial dysfunction, and oxidative stress ${ }^{12,18-20}$.

Regarding the presented evidence, OUA ICV injection in rats could be a candidate to a putative experimental model of BD by reproducing specific pathophysiological mechanisms of the disorder and simulate the related mood swings ${ }^{13,16}$. However, further tests are required for the validation of the model. Therefore, the present study was designed to evaluate the behavior and potential Hypothalamic-Pituitary-Adrenal (HPA) axis alteration seven, nine and fourteen days after a single OUA ICV administration in Wistar rats; Li effects in this experimental context were also evaluated.

\section{Materials and methods \\ Animals}

Sixty day old male Wistar rats (Rattus norvegicus) were provided by the animal house at University of Southern Santa Catarina (UNESC). Animals were placed five per cage in a room with controlled conditions (temperature: $22 \pm 1{ }^{\circ} \mathrm{C}$; relative humidity: $45-55 \%$ ), in a light/dark cycle of $12 / 12 \mathrm{~h}$ (lights at 0600 hours). Rats also received chow and water. Experiments were performed according to the Guide for the Care and Use of Laboratory Animals ${ }^{21}$ and the guidelines of Brazilian Society for Neuroscience and Behavior, with approval by the UNESC's Ethical Committee on Animal Use for Research (protocol \# 66/2010).

\section{Surgery and OUA administration}

Animals were submitted to anesthesia by intramuscular injection of ketamine and xylazine $(80 \mathrm{mg} / \mathrm{kg}$ and $10 \mathrm{mg} / \mathrm{kg}$ body weight, respectively). Thereafter, skin covering rat skull was removed and a 27 -gauge guide cannula $(9 \mathrm{~mm})$ was placed according to the following coordinates: posterior to bregma $(0.9 \mathrm{~mm})$; right from midline $(1.5 \mathrm{~mm})$; and above the lateral brain ventricle $(1.0 \mathrm{~mm})$. A $2 \mathrm{~mm}$ hole was made in the skull, through which a cannula was implanted ventrally to $2.6 \mathrm{~mm}$ of superior and fixed surface. On the fourth day after surgical procedure, animals received an ICV injection of artificial cerebrospinal fluid (aCSF) $5 \mu \mathrm{L}$ alone or in combination with OUA $\left(10^{-3} \mathrm{M}\right)^{14,17}$. A cannula (30gauge) was put inside guide cannula and linked to a microsyringe through a polyethylene tube. End of infusion cannula extended $1.0 \mathrm{~mm}$ beyond guide cannula aiming reach the right lateral brain ventricle.

\section{Experimental design}

According to Diagnostic and Statistical Manual of Mental Disorders (5th edition), in bipolar patients, manic episodes last at least 7 days, whereas depressive symptoms are present within a 14 days period in the same subjects ${ }^{22}$. Thus, open field test (manic-like behavior assessment), sweet food consumption test and forced swimming test (depressive-like behavior assessment) were conducted in three stages: 7, 9, and 14 days after OUA injection, in order to evaluate potential manic-like behavior in early stage and depressive-like behaviors in a later stage of the model.

To test the predictive validity, on the day following the ICV administration, animals received saline (sal) or $\mathrm{Li}$ $(47.5 \mathrm{mg} / \mathrm{kg})$ by intraperitoneal injections for 14 days, twice a day ${ }^{12}$. Thus the animals were divided by simple randomization in four groups (I) aCSF + sal; (II) aCSF + $\mathrm{Li}$; (III) OUA + sal; (IV) OUA + Li. $(n=8$ rats per group per stage $-7,9$, and 14 days. Sample size was calculated by resource equation method, this sample size is enough to get significant results):

\section{Behavioral tests}

The investigator were blinded to the group allocation during all the behavioral tests.

\section{Open field test}

In rodents, increased locomotor activity characterizes manic-like behavior ${ }^{23}$. Thus, locomotion was evaluated 7 or 14 days after ICV administrations through the open field test, according to the method described by Broadhurst $^{24}$.

\section{Forced swimming test}

Procedure was carried out 14 days after injections, according to standardized method ${ }^{25,26}$.

\section{Sweet food consumption}

Anhedonia was assessed according sweet food consumption $^{27}$. Experiment was conducted at 0800-1200 hours, 14 days following ICV administrations. 


\section{Biochemical analysis \\ Samples}

To test construct validity, at 7th, 9th, and 14th days after OUA injection, $24 \mathrm{~h}$ after last administration of $\mathrm{Li}$ or sal, blood and brain samples were collected to biochemical parameter analysis (descried bellow). Immediately after behavioral tests, blood samples of the anesthetized with ketamine and xylazine rats were collected at 0800-1200 hours by cardiac puncture. To obtain serum, blood was centrifuged at $3000 \times g$, during $5 \mathrm{~min}$, and then stored at $-70^{\circ} \mathrm{C}$ for subsequent analysis. Rats were submitted to euthanasia by decapitation immediately after collection of blood. The brain was excised and the cerebral structures (frontal cortex and hippocampus) were dissected, washed and stored at $-70^{\circ} \mathrm{C}$.

\section{Li levels in serum}

Li levels were determined in serum samples of Li groups animals $(\mathrm{aCSF}+\mathrm{Li}$ and $\mathrm{OUA}+\mathrm{Li})$ at the 7 th, 9th, and 14th days according to Chapoteau and coworkers ${ }^{28}$.

\section{$\mathrm{Na}^{+} / \mathrm{K}^{+}$-ATPase activity}

The reaction mixture for $\mathrm{Na}^{+} / \mathrm{K}^{+}$-ATPase assay contained $5.0 \mathrm{mM} \mathrm{MgCl} 2,80.0 \mathrm{mM} \mathrm{NaCl}, 20.0 \mathrm{~m} \mathrm{MKCl}$, and $40.0 \mathrm{mM}$ Tris- $\mathrm{HCl}, \mathrm{pH} 7.4$, in a final volume of $200 \mathrm{lL}$. The reaction was initiated by addition of ATP to a final concentration of $3.0 \mathrm{mM}$. Controls were carried out under the same conditions with the addition of $1.0 \mathrm{mM}$ ouabain. $\mathrm{Na}^{+} / \mathrm{K}^{+}$-ATPase activity was calculated by the difference between the two assays according to the method of Wyse and coworkers ${ }^{29}$. Released inorganic phosphate $(\mathrm{Pi})$ was measured by the method of Chan and coworkers ${ }^{30}$. Specific activity of the enzyme was expressed as nmol $\mathrm{Pi}$ released per min per mg of protein.

\section{Analysis of HPA axis parameters}

Adrenocorticotropic hormone (ACTH) and corticosterone levels were determined in serum samples of the three stages using radioimmunoassay-based kits developed by MP Biomedicals, LLC (Santa Ana, California, USA). Adrenal gland was excised and its weight then measured with a precision scale.

\section{Evaluation of oxidative stress parameters in the cerebral structures}

Lipid peroxidation Activity of lipid hydroperoxide (LPH) was evaluated using a specific kit provided by Cayman (Cayman Chemical Company, Ann Arbor, Michigan, USA; catalog no. 705003). Thiobarbituric acid reactive species (TBARS) Assay Kit (Cayman; catalog no. 10009055) was used for the direct quantitative measurement of the malondialdehyde (MDA) level in the samples of brain tissue. 4-Hydroxy-2-nonenal (4-HNE) content was quantified using the kit provided by Cell Biolabs (Cell Biolabs, Inc., San Diego, California, USA; catalog no. STA-338). 8-Isoprostane content was measured using the $\mathrm{ACE}^{\mathrm{TM}}$ Competitive EIAs Kit (Cayman; catalog no. 516351) with 8-isoprostane-acetylcholinesterase (EC 3.1.1.7) as a tracer and 8-isoprostane specific antiserum.

Protein adducts arising from modification of cysteine, histidine or lysine residues by 4-HNE were quantified with the method of Kimura and coworkers ${ }^{31}$.

Protein oxidation parameters Carbonyl group content was measured using a specific kit provided by Cell Biolabs (OxiSelect $^{\mathrm{TM}}$ Protein Carbonyl ELISA Kit; catalog no. STA310).

Activity of antioxidant enzymes Another important parameter evaluated was glutathione peroxidase (GPx, EC 1.11.1.9) activity. GPx catalyzes the reduction of peroxides coupled to glutathione oxidation. Oxidized glutathione is newly reduced in a NADPH-dependent process, and NADPH consumption is followed by spectrophotometry at $340 \mathrm{~nm}$ wavelength as described by Wendel ${ }^{32}$. Finally, glutathione reductase (GR, EC 1.8.1.7) activity was determined on the basis of NADPH oxidation at $340 \mathrm{~nm}$. Velocity of NADPH consumption reflects GR activity $^{33}$.

\section{Protein levels}

Protein content was measured to provide normalization for biochemical analysis, using as standard bovine serum albumin, according to method described by Lowry and coworkers $^{34}$.

\section{Statistical analysis}

Face validity results were evaluated by student's $t$ test and; Construct and predictive results were evaluated by Two-way analysis of variance (ANOVA) followed by Tukey's for detection of differences between groups. Data were presented as mean \pm standard deviation (SD). Software used in analysis was Statistica 7 (StatSoft, Inc., Tulsa, Oklahoma, USA). $p \leq 0.05$ was required to rate differences as statistically significant.

\section{Results}

Face validity of the animal model of BD induced by OUA

The face validity of an animal model of psychiatric disorder is the ability of the model in mimic the symptoms of the disorder. It can be observed that seven days after a single OUA administration, the animals presented manic-like behaviors, which were demonstrated through increased crossings, rearings and visits to the open-field center. In the ninth day after OUA ICV administration, 


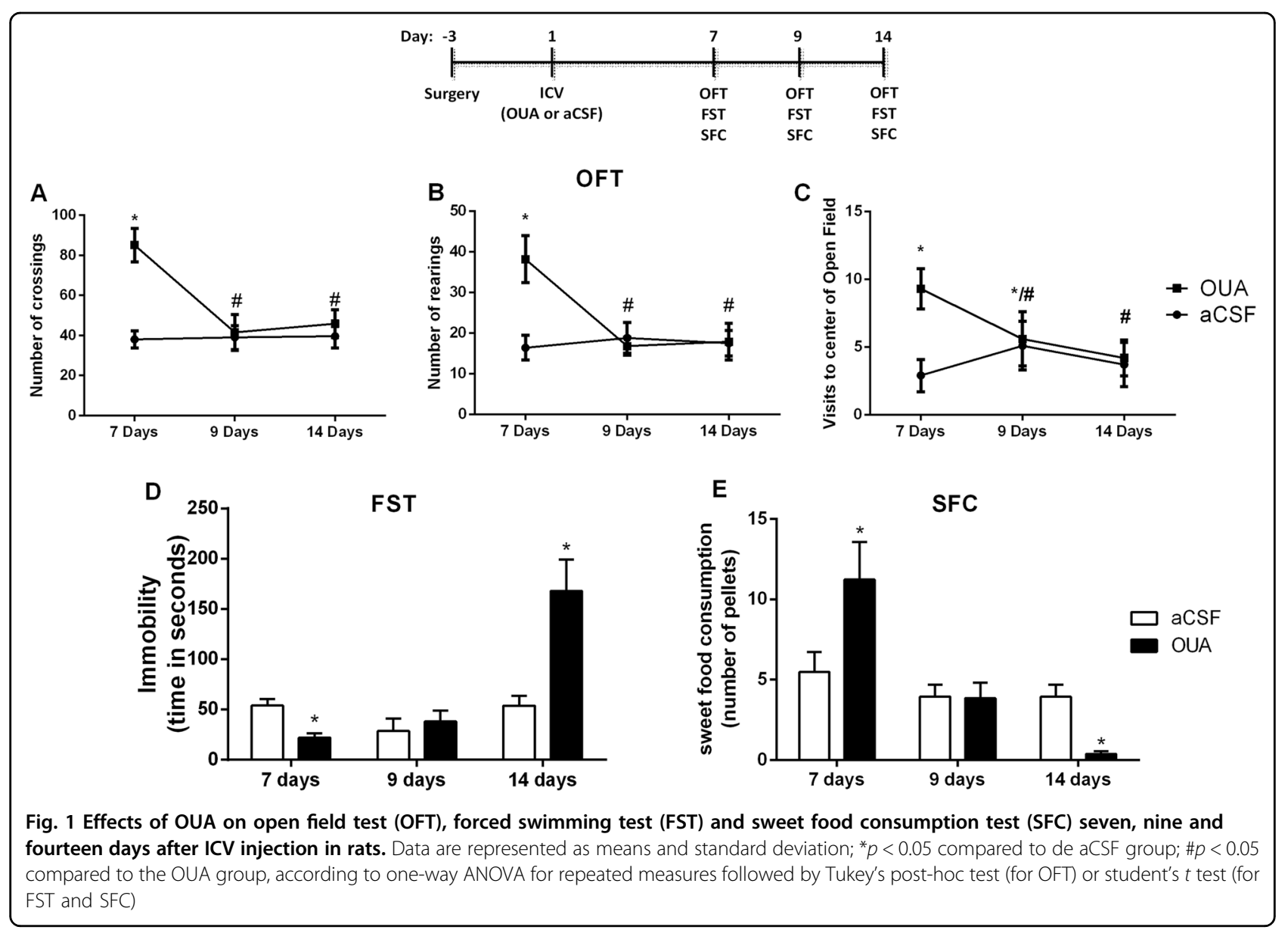

the same animals did not present locomotor (crossings), exploratory (rearings) or risk-taking behavior (visits to the open-field center) alterations (Fig. 1a-c). To evaluated depressive-like behavior, the animals were subjected to forced swimming and sweet food consumption tests (Fig. $1 d$, e). Nine days after OUA administration the animals did not present any behavioral alteration in the forced swimming or sweet food consumption tests when compared to the control group. Following the experimental design, fourteen days after OUA, the animals also did not show any alteration in the open-field test (Fig. 1a-c). However, fourteen days after OUA ICV injection, the animals increased the immobility time in the forced swimming test, which reflect a measure of behavioral despair. In the sweet food consumption test, a measurement of anhedonic-like behavior, rats OUA-administered reduced the sweet food pellet intake compared to the control group (Fig. 1d, e). It can be suggested that 14 days after OUA administration mimics depressive-like behaviors in rats. Together, these results demonstrate that a single injection of OUA induces manic- and depressivelike behaviors in the same animal, contemplating the face validity for a suitable animal model of BD.
Predictive validity of the animal model of $B D$ induced by OUA

The predictive validity of the animal model of psychiatric disorder is the ability of the model in mimic the treatment of the disorder. As described previously in the literature $^{13}$, Li reversed the increased in crossings, rearings, and visits to the open-field center induced by OUA seven days after ICV injection, mimicking the treatment of an acute manic episode (Fig. 2a-c). The rats treated with $\mathrm{Li}$ for nine days did not induce any behavioral alteration (open-field test, forced swimming and, sweet food consumption) after the ICV OUA injection, mimicking the maintenance of the treatment of $\mathrm{BD}$ (Fig. $2 \mathrm{a}-\mathrm{e})$. However, Li for nine days per se decreased the immobility time in the forced swimming test (Fig. 2d). Fourteen days of treatment with Li partially reversed the increased immobility time in the forced swimming test induced by OUA (Fig. 2d). Besides, Li reversed the decrease of food consumption induced by OUA (Fig. 2e), mimicking the treatment of a depressive episode. Therefore, it can be suggested that administration of $\mathrm{Li}$ in the OUA-administered rats mimics the treatment of bipolar disorder in both manic and depressive episodes, 


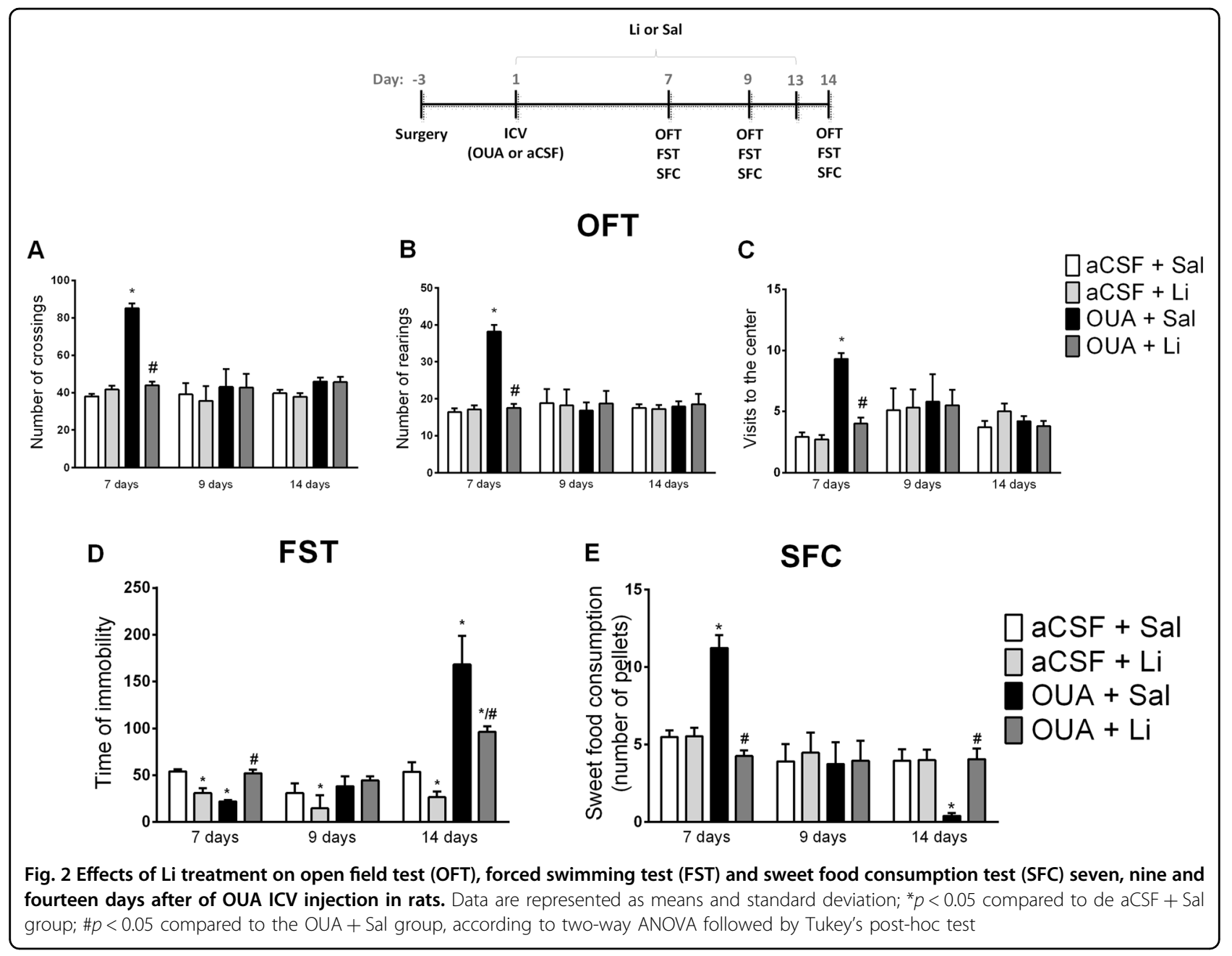

contemplating the predictive validity of the model. It is important to note that the plasmatic levels of $\mathrm{Li}$ of the animals treated with $\mathrm{Li}-7$, 9, and 14 days of treatment were between 0.8 and $1.2 \mathrm{meq} / \mathrm{L}$, which is the therapeutical dosage observed in bipolar patients (see Table 1).

\section{Construct validity of the animal model of BD induced by OUA}

The construct validity of the animal model of psychiatric disorder is the ability of the model in mimic some pathophysiologic alteration of the disorder. The development of the animal model of BD induced by OUA follow the hypothesis that decreased of $\mathrm{Na}^{+} / \mathrm{K}^{+}$-ATPase activity is the crucial factor in the trigger of manic and depressive mood episodes. Therefore, to confirm that the dose of OUA administered here decreases the $\mathrm{Na}^{+} / \mathrm{K}^{+}$ATPase activity, this enzyme activity was evaluated in the brain of rats. The $\mathrm{Na}^{+} / \mathrm{K}^{+}$-ATPase activity decreased in the frontal cortex of rats seven days after OUA administration (Fig. 3a). In the total brain, $\mathrm{Na}^{+} \mathrm{K}^{+}$-ATPase activity decreased seven and nine days after OUA administration (Fig. 3c). The treatment with Li reversed the $\mathrm{Na}^{+} \mathrm{K}^{+}$-ATPase inhibition induced by OUA (Fig. 3a, c). No alterations were observed on $\mathrm{Na}^{+} \mathrm{K}^{+}$-ATPase activity in the hippocampus of the animals (Fig. 3b). Together these results demonstrate that ICV administration of OUA has construct validate because decreased the $\mathrm{Na}^{+} / \mathrm{K}^{+}$-ATPase activity in the brains, as observed in bipolar patients.

To increase the construct validity of the $\mathrm{BD}$ animal model induced by OUA, we evaluated biological markers alterations observed in bipolar patients, such as HPA axis and oxidative stress parameters. Fourteen days after OUA administration increased the levels of ACTH (Fig. 3d) and corticosterone (Fig. 3e) and increased the weight of adrenal gland of the rats (Fig. 3f), demonstrating alterations in the HPA. The treatment with $\mathrm{Li}$ reversed all alterations in the HPA axis induced by OUA (Fig. $3 d-f$ ). The ACTH levels increased in the group OUA + Li nine days after ICV administration (Fig. 3d). No HPA axis alteration was observed seven days after OUA administration. 
Table 1 Lithium levels in serum of rats after 7, 9, and 14 days of OUA ICV injection

\begin{tabular}{|c|c|c|c|}
\hline & 7 days after & 9 days after & 14 days after \\
\hline \multirow{7}{*}{$\mathrm{aCSF}+\mathrm{Li}$} & 0.72 & 1.2 & 1.16 \\
\hline & 0.85 & 0.84 & 0.85 \\
\hline & 0.69 & 1.12 & 0.75 \\
\hline & 0.75 & 0.96 & 0.95 \\
\hline & 0.65 & 0.97 & 0.73 \\
\hline & 0.71 & 0.85 & 0.83 \\
\hline & 0.85 & 1.13 & 0.95 \\
\hline Mean \pm S.D. & $0.74 \pm 0.077$ & $1.01 \pm 0.14$ & $0.88 \pm 0.15$ \\
\hline \multirow[t]{7}{*}{ Ouabain \pm Li } & 0.92 & 1.12 & 0.74 \\
\hline & 0.78 & 0.97 & 0.75 \\
\hline & 0.69 & 0.92 & 1.12 \\
\hline & 0.85 & 0.85 & 0.85 \\
\hline & 0.96 & 0.96 & 0.75 \\
\hline & 0.75 & 0.87 & 1.1 \\
\hline & 0.81 & 0.85 & 0.95 \\
\hline Mean \pm S.D. & $0.82 \pm 0.095$ & $0.93 \pm 0.096$ & $0.89 \pm 0.16$ \\
\hline
\end{tabular}

Data are presented in absolute values and total mean \pm standard deviation

It is well described in the literature that bipolar patients have oxidative stress parameters altered in the brain; therefore, a suitable animal model of BD should present this biochemical alteration. In the present study, 7, 9, and 14 days after a single OUA administration induced an increase in the oxidative lipid damage in the frontal cortex and hippocampus of rats, which was evaluated through LPO (Fig. 4a, h), MDA (Fig. 4b, i), 4-HNE (Fig. $4 \mathrm{c}, \mathrm{j}$ ), and 8-ISO (Fig. 4d, k). An increase of protein carbonylation was observed in the frontal cortex and hippocampus of rats 7, 9, and 14 days after OUA administration (Fig. 4e, l). The treatment with Li reversed the oxidative damage to the lipid and protein induced by OUA, in both brain structures, and in all oxidative damage parameters evaluated (Fig. 4a-e, h-l). OUA ICV administration also alters antioxidant enzymes activities. Seven and fourteen days after ICV administration, OUA increased GR and GPx activities in frontal cortex and hippocampus, and Li reversed these enzymes alterations (Fig. 4f, g, m, n). However, 9 days after OUA administration increased the GPx activity in frontal cortex and hippocampus, but $\mathrm{Li}$ was not able to reverse this enzyme alteration. GR was not altered in any group nine days after ICV administration.

\section{Discussion}

Recent genetic, neurobiological and pharmacological advances have contributed to the development of new animal models, which have been essential tools to studying intracellular systems that may be involved in the pathophysiology of psychiatric disorders ${ }^{35,36}$. However, no animal model completely mimics a particular psychiatric disorder. The development of an animal model of BD is a real challenge for researchers because of the complicated clinical course, which involves episodes of mania and depression ${ }^{37}$. It is important to emphasize that, at the moment, no pharmacological animal model mimic mania and depression in the same animal. In the present study, it was demonstrated that a single ICV injection of OUA induced manic- and depressive-like behaviors, contemplating the face validity for the consolidation of the animal model of BD.

The present study showed significantly increased locomotor activity 7 days after OUA injection in rats. Indeed, some papers also reported manic-like behaviors following OUA ICV administration in rats, including increased locomotor and exploratory activities ${ }^{10,13,20,38}$. In contrast, depressive-like behaviors (increased immobility time and decreased sweet food consumption) were observed in the animals 14 days after they received OUA. Li and colleagues $^{16}$ demonstrated that OUA single injection significantly decreased motor activity in automated activity monitors, suggesting it as a model of bipolar depression. However, Li's study did not demonstrate manic-like behavior at the same animal; besides, hypolocomotion could not be considered the best depressive-like behavior parameter. Differently, the present study showed anhedonic- and hopelessness-like behaviors (considered as standard depressive-like parameters in animals) after a single OUA injection. Thus, this study is the first one to demonstrate OUA-induced opposite behaviors in the same animal.

Nine days after OUA administration the animals did not present any behavioral alteration in the open field, forced swimming and sweet food consumption tests when compared to the control group. Therefore, it can be suggested that 9 days after OUA injection in rats mimics a euthymic episode. In contrast, Ruktanonchai et $\mathrm{al}^{39}$. demonstrated OUA-induced hyperactivity in Sprague-Dawley rats persists for nine days. The discrepancy between the studies could be explained by the rat strains and experimental conditions differences. It is important to note that in the present study the protocol was repeated to biochemical analysis and we found the same in the open-field test, with no behavior alterations. The concept of euthymia does not include only the stable period with no mood alteration, but also an intra-state period when the patient do not present enough mood symptoms to be classified in a specific mood episode ${ }^{40}$. 


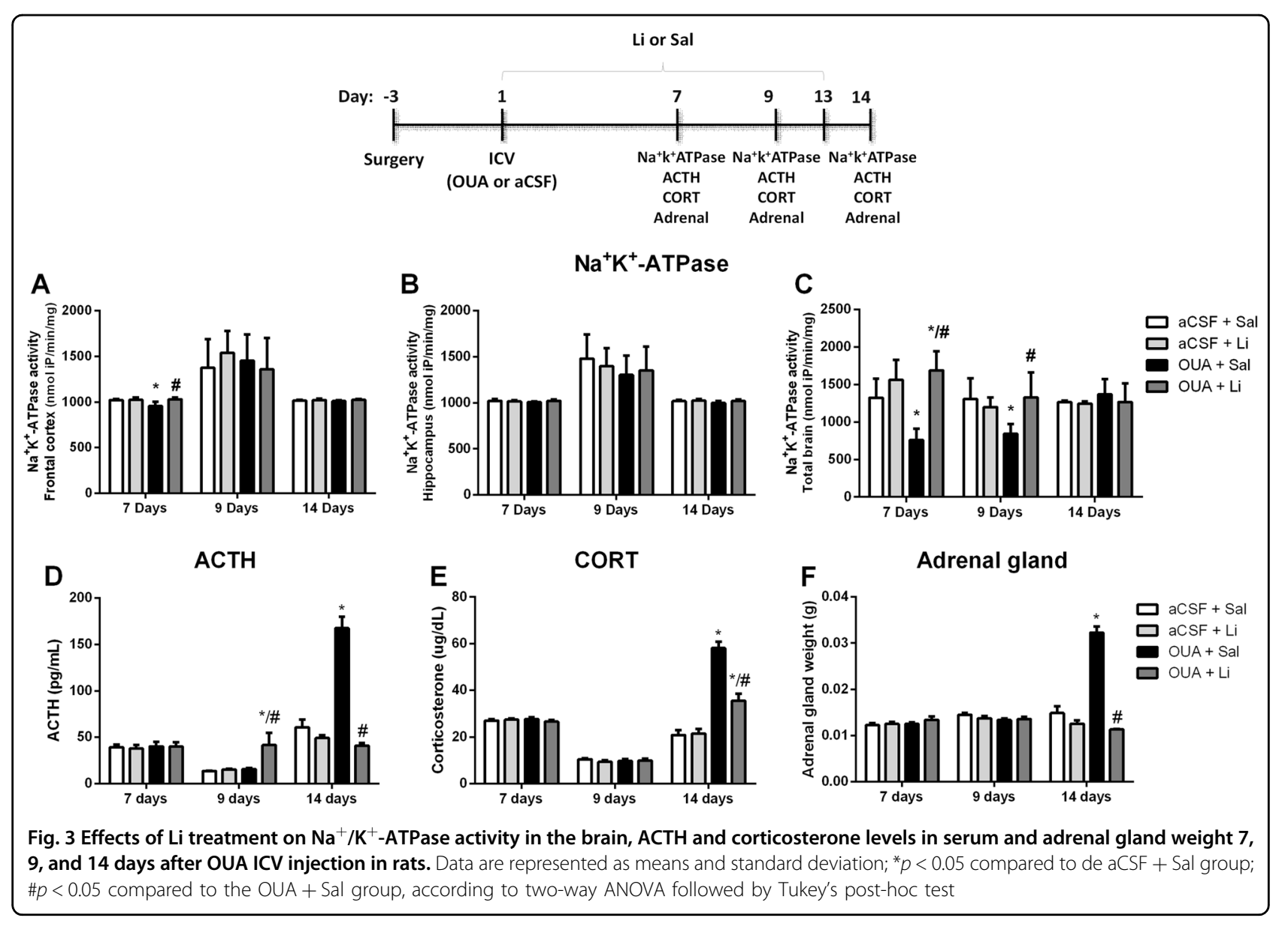

Thus, under our experimental conditions, it is possible to suggest that long-term OUA effects adequately fit the criteria of BD face validity, mimicking not only manic and depressive episodes but also an intra-state euthymia observed in BD patients.

The predictive validity of the animal model of psychiatric disorder is the ability of the model in mimic the treatment of the disorder. Li pretreatment for seven days prevented OUA-induced manic-like behaviors. There are several studies from our as well as other research groups demonstrating that $\mathrm{Li}$ treatment can reverse manic-like behavior in animals submitted to OUA ICV injection $^{10,13,20,38}$. Herein, Li administration partly reversed the immobility time and completely reversed decreasing in the sweet food intake. Although some preclinical studies have previously described $\mathrm{Li}$ antidepressant effects ${ }^{41,42}$, this is the first study mimicking the maintenance treatment of depressive- and manic-like behaviors in a potential BD animal model. These data suggest that ICV OUA administration in the rat may prove the predictive validity for a $\mathrm{BD}$ animal model.

The construct validity of the animal model of psychiatric disorder is the ability of the model in mimicking some pathophysiologic alteration of the disorder. The development of the animal model of $\mathrm{BD}$ induced by OUA follow the hypothesis that decreased of $\mathrm{Na}^{+} / \mathrm{K}^{+}$-ATPase activity is the crucial factor in the trigger of manic and depressive mood episodes. Therefore, to confirm that the dose of OUA administered here decreases the $\mathrm{Na}^{+} / \mathrm{K}^{+}$ATPase activity, this enzyme activity was evaluated in the brain of rats. It was observed that seven and nine days after ICV injection, OUA decreased $\mathrm{Na}^{+} / \mathrm{K}^{+}$-ATPase activity in the total brain of the animals. In the frontal cortex, the activity of this enzyme was decreased only seven days after OUA administration.

The hypothesis of $\mathrm{Na}^{+} / \mathrm{K}^{+}$-ATPase in the BD physiopathology was suggested more than 50 years ago ${ }^{43,44}$. Looney and El-mallakh ${ }^{45}$ demonstrated in a meta-analysis study that $\mathrm{Na}^{+} / \mathrm{K}^{+}$-ATPase activity is decreased in erythrocyte of BD patients. Since then, other studies keep contributing to this hypothesis ${ }^{46-49}$. Even a small reduction in this enzyme activity may lead the resting membrane potential close to threshold and then enhance neuronal excitability and impair $\mathrm{Ca}^{2+}$ depuration rate ${ }^{50,51}$. Increased neuronal excitability could trigger hyperactivity, which characterizes manic episodes in BD. However, 


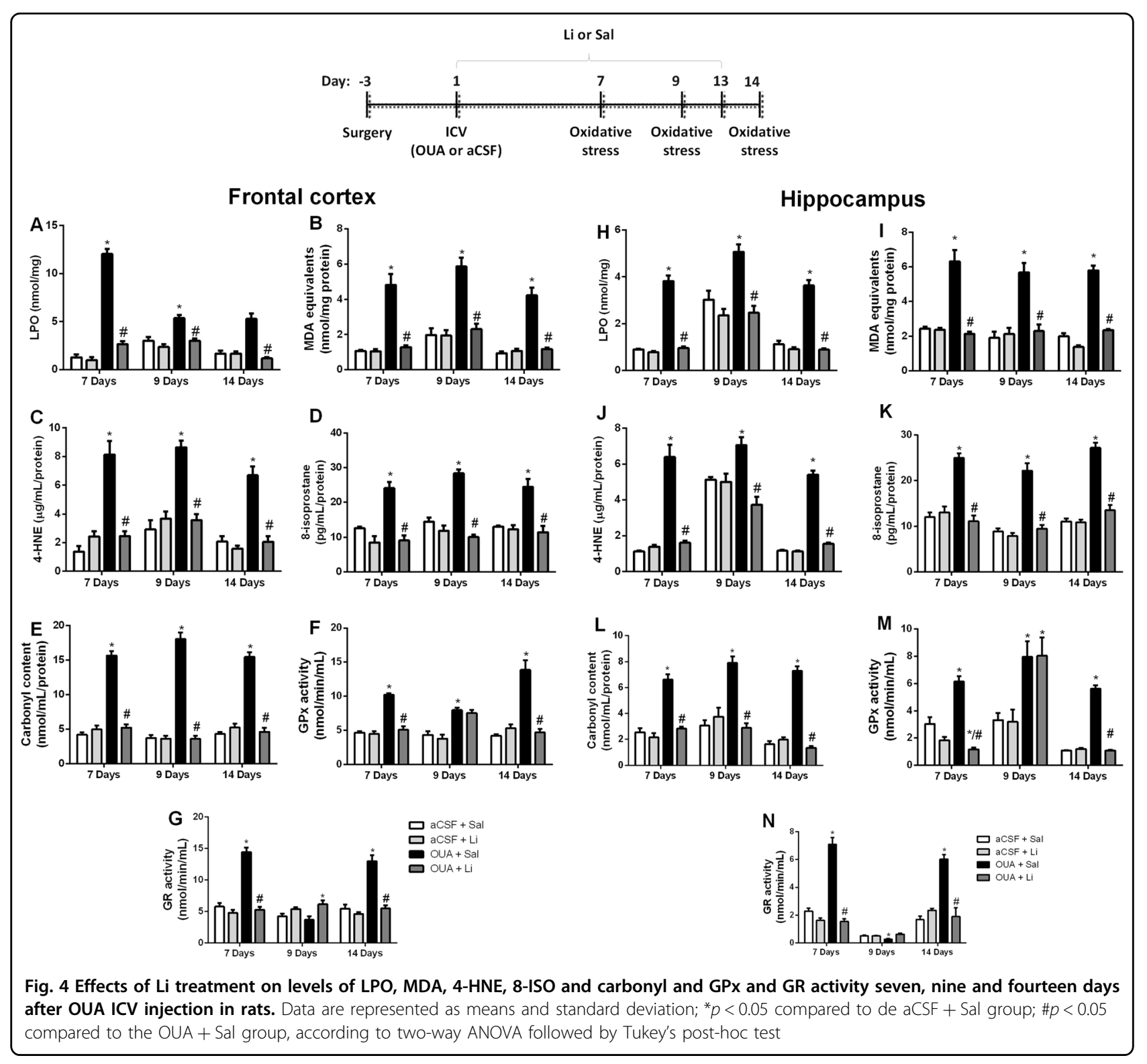

long-term $\mathrm{Na}^{+} / \mathrm{K}^{+}$-ATPase inhibition, which increases neuronal excitability could lead to decreases the control of resting potential, difficulting the following neuronal depolarization. All these events may reduce neuronal signaling velocity and, consequently, decrease synaptic efficiency of neurons, leading to depressive episodes of $\mathrm{BD}^{11,14}$. Together these studies highlight the involvement of this enzyme on $\mathrm{BD}$ pathophysiology and reinforcing its relevance as a construct validity to animal models of this disorder.

To increase the construct validity of the $\mathrm{BD}$ animal model induced by OUA, we evaluated biological markers alterations observed in bipolar patients, such as HPA axis and oxidative stress parameters. The pattern of depressed behavior elicited by OUA in rats was concomitant to increases in adrenal gland weight, corticosterone, and ACTH serum level. Enlargement of the hypophysis and adrenal gland due to deregulated secretion of cortisol and ACTH (HPA axis activation) was detected in depressive patients ${ }^{52-54}$. Similarly, animals submitted to maternal deprivation/chronic stress exhibit increases in the weight of adrenal gland and serum content of ACTH and corticosterone, mimicking physiological alterations of depressive disorders in these models ${ }^{55,56}$. HPA axis activation in animals receiving OUA is ascribed to increased ACTH and corticosterone levels and adrenal gland hypertrophy. Also, Li also abrogated increases in the ACTH levels and adrenal gland weight induced by OUA. Once again, these findings support the OUA animal model to the intended 
aim, in compliance with the construct, face and predictive validities required to a suitable animal model for $B D$.

Manic-, euthymic- and depressive-like behaviors were accompanied by increased oxidative damage to macromolecules from rat frontal cortex and hippocampus, 7, 9, or 14 days after OUA ICV injection. Data show increased content of LPH, MDA (TBARS), 4-HNE and 8-ISO in these cerebral structures. Increased protein oxidation was also detected in similar conditions. Li administration completely counteracted such oxidative disturbances. Previous observations of our research group also showed increased MDA and carbonyl levels in rat frontal cortex and hippocampus, 7 days after OUA administration ${ }^{13,19}$. Such alterations were significantly prevented by $\mathrm{Li}$ in the paper carried out by Jornada and coworkers.13 In addition, increased carbonyl, 3-nitrotyrosine, 4-HNE, and 8ISO contents were detected in the frontal cortex of BD patients in post mortem studies ${ }^{57-59}$. Significantly impaired $\mathrm{Na}+/ \mathrm{K}+-$ ATPase activity associated to lipid peroxidation in serum was also reported in BD patients, whereas these alterations were reversed by $\mathrm{Li}^{60}$. Thus, dysfunction of this enzyme activity probably is a pivotal mechanism in oxidative stress associated to the disorder. One of the therapeutic mechanisms of Li against oxidative damage could include enhancement of $\mathrm{Na}+/ \mathrm{K}$ + -ATPase activity. Data provided by the present paper show oxidative damage in rats receiving OUA, mimicking pathophysiological aspects in BD patients.

The present study also showed increased activity of GPx and GR in cerebral structures of rats receiving OUA, during the manic- and depressive-like behaviors; $\mathrm{Li}$ administration reversed these enzyme alterations. Partly in line with such observations, Andreazza and colleagues $^{61}$ detected an increase in GR activity in blood of BD patients at late stages of the disorder. Besides, therapy with the glutathione precursor $\mathrm{N}$-acetylcysteine in $\mathrm{BD}$ patients alleviated their manic and depressive symptoms $^{62}$. Indeed, animal models of mania and depression have reported altered activity of antioxidant glutathione enzymes in the brain. To illustrate, a paper carried out using a depression model induced by immobilization stress showed increased activity of these enzymes in the hippocampus and cerebral cortex of rodents ${ }^{63}$, indicating that models of depression induce alterations in antioxidant enzymes resembling to that reported in the present study. However, Brocardo and coworkers ${ }^{64}$ showed decreased activity of GPx and GR in the same cerebral structures assessed here soon after OUA ICV injection in rats. Such discrepancy may be attributed to differences in the experimental designs between papers because we measured the activity of the antioxidant enzymes 7 or 14 days after OUA administration, aiming to simulate late stages of BD. Li administration prevented the deleterious modifications in GPx and GR elicited by OUA in cerebral structures of rats. Li was also implicated in the modulation of these enzyme activities in the brain of rats receiving amphetamine - a classic model of mania, potentially preventing oxidative damages in these animals ${ }^{65}$. Presented evidence hints alteration in the GPx and GR activities as a central factor for BD pathophysiology. One of the putative therapeutic roles of $\mathrm{Li}$ includes modulation of these antioxidant enzymes, collaborating to the maintenance of redox homeostasis in the brain.

Therefore, the OUA animal model comply with the three primary criteria (validities) required an adequate BD model. Since studies reported a close interaction between HPA axis and oxidative stress in BD patients ${ }^{66,67}$, reduced activity of $\mathrm{Na}^{+} / \mathrm{K}^{+}$-ATPase detected in these patients could be linked to increased secretion of glucocorticoid hormones and oxidative damage, leading to the mood swings. As a mood stabilizer drug, Li acts counteracting these pathological changes, which contributes to mitigating BD symptoms. Proposed OUA model could be used in studies on the pathophysiology of the disorder and to the screening of promising mood stabilizers drug candidates.

\begin{abstract}
Acknowledgements
Translational Psychiatry Program (USA) is supported by the Department of Psychiatry and Behavioral Sciences, McGovern Medical School, The University of Texas Health Science Center at Houston (UTHealth). Translational Psychiatry Laboratory (Brazil) is one of the centers of the National Institute for Molecular Medicine (INCT-MM) and one of the members of the Center of Excellence in Applied Neurosciences of Santa Catarina (NENASC). Its research is promoted by grants from CNPq, CAPES (Finance code 001), FAPESC, Instituto Cérebro e Mente and UNESC. João Quevedo is a 1A CNPq Research Fellow. We thank to Dr. L Trevor Young and Dr. Ana Cristina Andreazza for support with oxidative stress parameters analysis at University of Toronto.
\end{abstract}

\section{Author details \\ ${ }^{1}$ Translational Psychiatry Laboratory, Graduate Program in Health Sciences, University of Southern Santa Catarina (UNESC), Criciúma, SC, Brazil. \\ ${ }^{2}$ Translational Psychiatry Program, Department of Psychiatry and Behavioral Sciences, McGovern Medical School, The University of Texas Health Science Center at Houston (UTHealth), Houston, TX, USA. ${ }^{3}$ Center of Excellence on Mood Disorders, Department of Psychiatry and Behavioral Sciences, McGovern Medical School, The University of Texas Health Science Center at Houston (UTHealth), Houston, TX, USA. ${ }^{4}$ Neuroscience Graduate Program, The University of Texas MD Anderson Cancer Center UTHealth Graduate School of Biomedical Sciences, Houston, TX, USA}

\section{Conflict of interest}

J.Q. has the following declarations of interest: Clinical Research Support: Janssen Pharmaceutical (Clinical Trial), Allergan (Clinical Trial); Advisory Boards, Speaker Bureaus, Expert Witness, or Consultant: Daiichi Sankyo (Speaker Bureau); Patent, Equity, or Royalty: Instituto de Neurociencias Dr. Joao Quevedo (Stockholder); Other: Artmed Editora (Copyright), Artmed Panamericana (Copyright). The remaining authors declare that they have no conflict of interest.

\section{Publisher's note}

Springer Nature remains neutral with regard to jurisdictional claims in published maps and institutional affiliations. 
Received: 11 April 2019 Accepted: 29 April 2019

Published online: 04 June 2019

\section{References}

1. Maletic, V. \& Raison, C. Integrated neurobiology of bipolar disorder. Front. Psychiatry 5, 98 (2014).

2. Naaldijk, Y. M., Bittencourt, M. C., Sack, U. \& Ulrich, H. Kinins and microglial responses in bipolar disorder: a neuroinflammation hypothesis. Biol. Chem. 397, 283-296 (2016).

3. Saxena, A. et al. Role of protein kinase $\mathrm{C}$ in bipolar disorder: a review of the current literature. Mol. Neuropsychiatry 3, 108-124 (2017).

4. Kato, T., Kasahara, T., Kubota-Sakashita, M., Kato, T. M. \& Nakajima, K. Animal models of recurrent or bipolar depression. Neuroscience 321, 189-196 (2016).

5. Logan, R. W. \& McClung, C. A. Animal models of bipolar mania: the past, present and future. Neuroscience 321, 163-188 (2016).

6. Sharma, A. N. et al. Modeling mania in preclinical settings: a comprehensive review. Prog. Neuropsychopharmacol. Biol. Psychiatry 66, 22-34 (2016).

7. Valvassori, S. S., Budni, J., Varela, R. B. \& Quevedo, J. Contributions of animal models to the study of mood disorders. Rev. Bras. Psiquiatr 35(Suppl 2), S121-S131 (2013).

8. Machado-Vieira, R., Kapczinski, F. \& Soares, J. C. Perspectives for the development of animal models of bipolar disorder. Prog. Neuropsychopharmacol. Biol. Psychiatry 28, 209-224 (2004).

9. Ellenbroek, B. A. \& Cools, A. R. Animal models with construct validity for schizophrenia. Behav. Pharm. 1, 469-490 (1990).

10. El-Mallakh, R. S. et al. Intracerebroventricular administration of ouabain as a model of mania in rats. Bipolar Disord. 5, 362-365 (2003).

11. Herman, L., Hougland, T. \& El-Mallakh, R. S. Mimicking human bipolar ion dysregulation models mania in rats. Neurosci. Biobehav. Rev. 31, 874-881 (2007).

12. Jornada, L. K. et al. Effects of mood stabilizers on hippocampus and amygdala BDNF levels in an animal model of mania induced by ouabain. J. Psychiatr. Res. 44, 506-510 (2010).

13. Jornada, L. K. et al. Lithium and valproate modulate antioxidant enzymes and prevent ouabain-induced oxidative damage in an animal model of mania. J. Psychiatr. Res. 45, 162-168 (2011).

14. El-Mallakh, R. S. \& Wyatt, R. J. The Na,K-ATPase hypothesis for bipolar illness. Biol. Psychiatry 37, 235-244 (1995).

15. Valvassori, S. S. et al. Effects of mood stabilizers on oxidative stress-induced cell death signaling pathways in the brains of rats subjected to the ouabaininduced animal model of mania: mood stabilizers exert protective effects against ouabain-induced activation of the cell death pathway. J. Psychiatr. Res. 65, 63-70 (2015).

16. Li, R., El-Mallakh, R. S., Harrison, L., Changaris, D. G. \& Levy, R. S. Lithium prevents ouabain-induced behavioral changes. Toward an animal model for manic depression. Mol. Chem. Neuropathol. 31, 65-72 (1997).

17. El-Mallakh, R. S. The Na,K-ATPase hypothesis for manic-depression. I. General considerations. Med. Hypotheses 12, 253-268 (1983).

18. Riegel, R. E. et al. Animal model of mania induced by ouabain: evidence of oxidative stress in submitochondrial particles of the rat brain. Neurochem. Int 55, 491-495 (2009).

19. Riegel, R. E. et al. Intracerebroventricular ouabain administration induces oxidative stress in the rat brain. Int J. Dev. Neurosci. 28, 233-237 (2010).

20. Lopes-Borges, J. et al. Histone deacetylase inhibitors reverse manic-like behaviors and protect the rat brain from energetic metabolic alterations induced by ouabain. Pharm. Biochem Behav. 128, 89-95 (2015).

21. National Research Council (US) Committee for the Update of the Guide for the Care and Use of Laboratory Animals. Guide for the Care and Use of Laboratory Animals 8th edn (National Academies Press, Washington (DC), 2011).

22. American Psychiatric Association. Diagnostic and Statistical Manual of Mental Disorders 5th edn, 124-125 (Amer Psychiatric Pub Incorporated, Washington, DC, 2013).

23. Nestler, E. J. \& Hyman, S. E. Animal models of neuropsychiatric disorders. Nat. Neurosci. 13, 1161-1169 (2010).

24. Broadhurst, P. L. in Experiments in Personality. (ed Eysenck, H. J.) 31-71 (Routledge \& Kegan Paul, London, 1960).

25. Porsolt, R. D., Le Pichon, M. \& Jalfre, M. Depression: a new animal model sensitive to antidepressant treatments. Nature 266, 730-732 (1977).
26. Detke, M. J., Rickels, M. \& Lucki, I. Active behaviors in the rat forced swimming test differentially produced by serotonergic and noradrenergic antidepressants. Psychopharmacology 121, 66-72 (1995).

27. Gamaro, G. D., Manoli, L. P., Torres, I. L., Silveira, R. \& Dalmaz, C. Effects of chronic variate stress on feeding behavior and on monoamine levels in different rat brain structures. Neurochem. Int. 42, 107-114 (2003).

28. Chapoteau, E., Czech, B. P., Zazulak, W. \& Kumar, A. First practical colorimetric assay of lithium in serum. Clin. Chem. 38, 1654-1657 (1992).

29. Wyse, A. T. et al. Methylmalonate administration decreases $\mathrm{Na}^{+}, \mathrm{K}^{+}$-ATPase activity in cerebral cortex of rats. Neuroreport 11, 2331-2334 (2000).

30. Chan, K. M., Delfert, D. \& Junger, K. D. A direct colorimetric assay for $\mathrm{Ca}^{2}$ ${ }^{+}$-stimulated ATPase activity. Anal. Biochem. 157, 375-380 (1986).

31. Kimura, $\mathrm{H}$. et al. Rapid increase in serum lipid peroxide 4-hydroxynonenal (HNE) through monocyte NADPH oxidase in early endo-toxemia. Free Radic. Res. 39, 845-851 (2005).

32. Wendel, A. Glutathione peroxidase. Methods Enzym. 77, 325-333 (1981).

33. Carlberg, I. \& Mannervik, B. Glutathione reductase. Methods Enzym. 113, 484-490 (1985)

34. Lowry, O. H., Rosebrough, N. J., Farr, A. L. \& Randall, R. J. Protein measurement with the Folin phenol reagent. J. Biol. Chem. 193, 265-275 (1951).

35. Manji, H. K. \& Chen, G. PKC, MAP kinases and the bcl-2 family of proteins as long-term targets for mood stabilizers. Mol. Psychiatry 7(Suppl 1), S46-S56 (2002).

36. Einat, H., Belmaker, R. H. \& Manii, H. K. New approaches to modeling bipolar disorder. Psychopharmacol. Bull. 37, 47-63 (2003).

37. Malkesman, O, Austin, D. R. Chen, G. \& Manii, H. K. Reverse translational strategies for developing animal models of bipolar disorder. Dis. Model Mech. 2, 238-245 (2009).

38. Valvassori, S. S. et al. Lithium and valproate act on the GSK-3 $\beta$ signaling pathway to reverse manic-like behavior in an animal model of mania induced by ouabain. Neuropharmacology 117, 447-459 (2017).

39. Ruktanonchai, D. J., El-Mallakh, R. S., Li, R. \& Levy, R. S. Persistent hyperactivity following a single intracerebroventricular dose of ouabain. Physiol. Behav. 63, 403-406 (1998).

40. Fava, G. A. \& Bech, P. The concept of euthymia. Psychother. Psychosom. 85, 1-5 (2016).

41. Silva, R. et al. Lithium blocks stress-induced changes in depressive-like behavior and hippocampal cell fate: the role of glycogen-synthase-kinase-3beta. Neuroscience 152, 656-669 (2008).

42. Mohseni, G. et al. Agmatine enhances the antidepressant-like effect of lithium in mouse forced swimming testthrough NMDA pathway. Biomed. Pharmacother. 88, 931-938 (2017).

43. Shaw, D. M. Mineral metabolism, mania, and melancholia. Br. Med. J. 2 262-267 (1966).

44. Coppen, A., Shaw, D. M., Malleson, A. \& Costain, R. Mineral metabolism in mania. Br. Med. J. 1, 71-75 (1966)

45. Looney, S. W. \& El-Mallakh, R. S. Meta-analysis of erythrocyte Na,K-ATPase activity in bipolar illness. Depress Anxiety 5, 53-65 (1997).

46. Goldstein, I. et al. Association between sodium- and potassium-activated adenosine triphosphatase alpha isoforms and bipolar disorders. Biol. Psychiatry 65, 985-991 (2009).

47. Kurup, A. R. \& Kurup, P. A. Membrane $\mathrm{Na}(+)-\mathrm{K}+$ ATPase mediated cascade in bipolar mood disorder, major depressive disorder, and schizophrenia-relationship to hemispheric dominance. Int. J. Neurosci. 112, 965-982 (2002)

48. Mynett-Johnson, L. et al. Evidence for an allelic association between bipolar disorder and a $\mathrm{Na}+\mathrm{K}+$ adenosine triphosphatase alpha subunit gene (ATP1A3). Biol. Psychiatry 44, 47-51 (1998).

49. Rose, A. M. et al. Alpha 2 isoform of the Na,K-adenosine triphosphatase is reduced in temporal cortex of bipolar individuals. Biol. Psychiatry 44, 892-897 (1998).

50. Martin, A. R. Quantal nature of synaptic transmission. Physiol. Rev. 46, 51-56 (1966).

51. McCarren, M. \& Alger, B. E. Sodium-potassium pump inhibitors increase neuronal excitability in the rat hippocampal slice: role of a $\mathrm{Ca}^{2+}$-dependent conductance. J. Neurophysiol. 57, 496-509 (1987).

52. Gold, P. W., Goodwin, F. K. \& Chrousos, G. P. Clinical and biochemical manifestations of depression. Relation to the neurobiology of stress. N. Engl. J. Med. 319, 413-420 (1988)

53. de Kloet, E. R., Joëls, M. \& Holsboer, F. Stress and the brain: from adaptation to disease. Nat. Rev. Neurosci. 6, 463-475 (2005). 
54. Juruena, M. F. Early-life stress and HPA axis trigger recurrent adulthood depression. Epilepsy Behav. 38, 148-159 (2014).

55. Owens, M. J. \& Nemeroff, C. B. The role of corticotropin-releasing factor in the pathophysiology of affective and anxiety disorders: laboratory and clinical studies. Ciba Found. Symp. 172, 296-308 (1993).

56. Oomen, C. A. et al. Opposite effects of early maternal deprivation on neurogenesis in male versus female rats. PLOS ONE 4, 3675-3680 (2009).

57. Wang, J. F., Shao, L., Sun, X. \& Young, L. T. Increased oxidative stress in the anterior cingulate cortex of subjects with bipolar disorder and schizophrenia. Bipolar Disord. 11, 523-529 (2009).

58. Andreazza, A. C., Shao, L., Wang, J. F. \& Young, L. T. Mitochondrial complex I activity and oxidative damage to mitochondrial proteins in the prefrontal cortex of patients with bipolar disorder. Arch. Gen. Psychiatry 67, 360-368 (2010).

59. Andreazza, A. C., Wang, J. F., Salmasi, F., Shao, L. \& Young, L. T. Specific subcellular changes in oxidative stress in prefrontal cortex from patients with bipolar disorder. J. Neurochem. 127, 552-561 (2013).

60. Banerjee, U., Dasgupta, A., Rout, J. K. \& Singh, O. P. Effects of lithium therapy on $\mathrm{Na}^{+}-\mathrm{K}^{+}$-ATPase activity and lipid peroxidation in bipolar disorder. Prog. Neuropsychopharmacol. Biol. Psychiatry 37, 56-61 (2012).
61. Andreazza, A. C. et al. 3-Nitrotyrosine and glutathione antioxidant system in patients in the early and late stages of bipolar disorder. J. Psychiatry Neurosci. 34, 263-271 (2009).

62. Magalhães, P. V. et al. A preliminary investigation on the efficacy of $N$-acetyl cysteine for mania or hypomania. Aust. N. Z. J. Psychiatry 47, 564-568 (2013).

63. Budni, J. et al. Folic acid prevents depressive-like behavior and hippocampal antioxidant imbalance induced by restraint stress in mice. Exp. Neurol. 240, 112-121 (2013).

64. Brocardo, P. S. et al. Folic acid administration prevents ouabain-induced hyperlocomotion and alterations in oxidative stress markers in the rat brain. Bipolar Disord. 12, 414-424 (2010).

65. Macêdo, D. S. et al. Effects of lithium on oxidative stress and behavioral alterations induced by lisdexamfetamine dimesylate: relevance as an animal model of mania. Prog. Neuropsychopharmacol. Biol. Psychiatry 43, 230-237 (2013).

66. Muneer, A. The neurobiology of bipolar disorder: an integrated approach. Chonnam Med. J. 52, 18-37 (2016)

67. Sigitova, E., Fišar, Z., Hroudová, J., Cikánková, T. \& Raboch, J. Biological hypotheses and biomarkers of bipolar disorder. Psychiatry Clin. Neurosci. 71, 77-103 (2017). 